\title{
INVERSE SEMIGROUPS OF HOMEOMORPHISMS BETWEEN OPEN SUBSETS
}

\author{
BRIDGET BOS BAIRD \\ (Received 27 April 1976) \\ Communicated by T. E. Hall
}

\begin{abstract}
The symbol $\mathscr{I}_{G}(X)$ denotes the inverse semigroup, under composition of functions. of all homeomorphisms between open subsets of a $T_{1}$ topological space $X$. The first result is that two such semigroups $\mathscr{I}_{G}(X)$ and $\mathscr{I}_{G}(Y)$ are isomorphic if and only if the spaces $X$ and $Y$ are homeomorphic. Ideals of $\mathscr{I}_{G}(X)$ are next examined and it is shown that for many spaces $X$ the semigroup $\mathscr{I}_{G i}(X)$ is 0 -simple. We also look at congruences on $\mathscr{I}_{G i}(X)$; one result is that we determine a congruence which in many instances is the largest proper congruence on $\mathscr{I}_{G}(X)$.
\end{abstract}

\section{Introduction}

If $X$ is a nonempty topological space then the set of all homeomorphisms whose domain and range are open subsets of $X$ forms a semigroup under composition of functions; we denote it by $\mathscr{I}_{G}(X)$. In fact this semigroup is an inverse semigroup (for every element $a$ in the semigroup there exists a unique element $b$, called the inverse of $a$, such that $a b a=a$ and $b a b=b) . \mathscr{I}_{G}(X)$ is a subsemigroup of the symmetric inverse semigroup $\mathscr{I}_{X}$ (the inverse semigroup, under composition, of all injective partial maps on the set $X$ ) and consists of all maps which preserve the topological structure of $X$ and have as domain and range open subsets of $X$. The empty map, denoted by 0 , belongs to $\mathscr{I}_{G}(X)$. We will consider $\mathscr{I}_{G}(X)$ for topological $T_{1}$ spaces $X$.

The first result is an isomorphism theorem: if $\mathscr{I}_{G}(X)$ and $\mathscr{I}_{G}(Y)$ are isomorphic then $X$ and $Y$ are homeomorphic (the converse clearly holds). The next section of the paper examines ideals of $\mathscr{I}_{G}(X)$. It turns out that for many spaces $X$, including $R^{n}$ (the cartesian product of $n$ copies of the real line), the rationals, and the Cantor discontinuum, the semigroup $\mathscr{I}_{G}(X)$ is

The results in this paper are from the author's doctoral dissertation, written at the State University of New York at Buffalo under the direction of K. D. Magill. Jr. 
0 -simple (the only ideals are $\mathscr{I}_{G}(X)$ and $\{0\}$ ). This is not always the case, however, and as an example the ideals of $\mathscr{I}_{G}(I)$ are enumerated ( $I$ denotes the closed unit interval). The last section examines congruences on $\mathscr{I}_{G}(X)$ for $T_{2}$ spaces $X$. If $\mathscr{I}_{G}(X)$ is 0 -simple then the largest proper congruence on $\mathscr{I}_{G}(X)$ is given; in other instances a minimal proper congruence on $\mathscr{I}_{G}(X)$ is determined. Another result of this section shows that the only idempotent separating congruence on $\mathscr{I}_{G}(X)$ is the diagonal congruence.

General information about semigroups will be from Clifford \& Preston (1961, 1967). The notations dom $f$ and $\operatorname{ran} f$ will denote the domain and the range of a function $f$. If $f, g \in \mathscr{I}_{G}(X)$ and $\operatorname{dom} f \cap \operatorname{ran} g=\varnothing$ then $f \circ g=0$ (the empty map). It is clear that 0 is the zero of $\mathscr{I}_{G}(X)$. If $f \in \mathscr{I}_{G}(X)$ then the inverse of $f$ (in the semigroup) is just the inverse mapping $f^{-1}$. Idempotents (elements $f$ such that $f \circ f=f$ ) are identity maps on open subsets $U$ of $X$ and will be denoted by $i_{U}$.

\section{Isomorphism theorem for $\mathscr{I}_{G}(X)$}

THEOREM 2.1. There exists an isomorphism $\varphi$ from $\mathscr{I}_{G}(X)$ onto $\mathscr{I}_{G}(Y)$ if and only if there exists a homeomorphism $h$ from $X$ onto $Y$. If $\varphi$ is such an isomorphism then $h$ can be chosen so that $\varphi(f)=h \circ f \circ h^{-1}$ for all $f \in \mathscr{I}_{G}(X)$. Conversely, if $h$ is a homeomorphism from $X$ onto $Y$, then $\varphi$, defined by $\varphi(f)=h \circ f \circ h^{-1}$ for $f \in \mathscr{I}_{G}(X)$, is an isomorphism from $\mathscr{I}_{G}(X)$ onto $\mathscr{I}_{G}(Y)$.

Proof. Suppose $\varphi$ is an isomorphism from $\mathscr{I}_{G}(X)$ onto $\mathscr{I}_{G}(Y)$. If $X$ has one point then the conclusion immediately follows $\left(0\right.$ and $i_{x}$ are the only elements in $\left.\mathscr{I}_{G}(X)\right)$. So now suppose $X$ has more than one point and let $x \in X$. Since $X$ is a $T_{1}$ space the set $X-\{x\}$ is a nonempty open subset of $X$. Homomorphisms of semigroups carry idempotents onto idempotents and so $\varphi\left(i_{x-\{x\}}\right)=i_{V}$ where $V$ is open in $Y$. Since $\varphi$ is an isomorphism we have that $V \neq Y$ and $V \neq \varnothing$. Suppose $y \notin V$. Then $V \subseteq Y-\{y\}$ and if $\varphi^{-1}\left(i_{Y-\{y\}}\right)=i_{U}$ then

$$
\begin{aligned}
i_{X-\{X\}} & =\varphi^{-1}\left(i_{V}\right)=\varphi^{-1}\left(i_{V \cap(Y-\{y\}}\right)=\varphi^{-1}\left(i_{V} \circ i_{Y-\{y\}}\right)=\varphi^{-1}\left(i_{V}\right) \circ \varphi^{-1}\left(i_{Y<\{y\}}\right) \\
& \left.=i_{X-\{x\}} \circ i_{U}=i_{U \cap(X-\{x\}}\right) .
\end{aligned}
$$

Hence $X-\{x\} \subseteq U$. Since $Y-\{y\} \neq Y$ we have that $U \neq X$. Thus $X-\{x\}=$ $U$ and so $V=Y-\{y\}$. Define a map $h$ from $X$ into $Y$ by $h(x)=y$. Then $\varphi\left(i_{X-\{x\}}\right)=i_{Y-\{h(x)\}}$.

If $x \neq x^{\prime}$ then $X-\{x\} \neq X-\left\{x^{\prime}\right\}$ and so $h(x) \neq h\left(x^{\prime}\right)$. Thus $h$ is injective. The above reasoning applied to $\varphi^{-1}$ yields that $\varphi^{-1}\left(i_{Y-\{y\}}\right)=i_{X-\{x\}}$ for some $x \in X$ and so $\operatorname{ran} h=Y$. Thus $h$ is a bijection from $X$ onto $Y$.

Next we show that $\varphi\left(i_{U}\right)=i_{h(U)}$ for all $U$ open in $X$, and $\varphi^{-1}\left(i_{V}\right)=i_{h}^{-1}(V)$ 
for all $V$ open in $Y$. If $\varphi\left(i_{U}\right)=i_{w}$ and $x \in U$ then $U$ is not contained in $X-\{x\}$ and so $W$ is not contained in $Y-\{h(x)\}$. Hence $h(x) \in W$ and so $h(U) \subseteq W$. Conversely, if $y \in W$ then $W$ is not contained in $Y-\{y\}$ and so $U$ is not contained in $X-\left\{h{ }^{\prime}(y)\right\}$. Thus $h{ }^{\prime}(y) \in U$ and so $y \in h(U)$. Hence $h(U)=W$ and $\varphi\left(i_{U}\right)=i_{h(U)}$. Now suppose $V$ is open in $Y$ and $\varphi^{\prime}\left(i_{V}\right)=i_{G}$. Then $h(G)=V$ and since $h$ is injective we have that $G=h$ ' $(V)$. Thus $\varphi^{-1}\left(i_{v}\right)=i_{h}{ }^{\prime}(1)$.

Now we show that $h$ is a homeomorphism. If $V$ is open in $Y$ then $\varphi{ }^{\prime}\left(i_{V}\right)=i_{h}{ }^{\prime}(v)$ and since $i_{h}{ }^{\prime}(v) \in \mathscr{I}_{G}(X)$ this means that $h{ }^{\prime}(V)$ is open. Thus $h$ is continuous. Likewise $h$ is open.

To complete this part of the proof we must show that if $f \in \mathscr{I}_{G}(X)$ then $\varphi(f)=h \circ f \circ h^{-1}$. If $f \in \mathscr{I}_{G}(X)$ and $U=\operatorname{dom} f$ then

$$
\operatorname{dom} \varphi(f)=\operatorname{dom} \varphi\left(f^{\prime \prime} \circ f\right)=\operatorname{dom} \varphi\left(i_{U}\right)=h(U)=\operatorname{dom}\left(h \circ f \circ h^{1}\right) .
$$

Likewise $\operatorname{ran} \varphi(f)=h(\operatorname{ran} f)$. Now let $y \in \operatorname{dom} \varphi(f)$. Then $y=h(x)$ where $x \in \operatorname{dom} f$. Let $G$ be any open neighborhood of the point $x$ which is contained in $\operatorname{dom} f$. Then $f \circ i_{G} \in \mathscr{I}_{G}(X)$ with $\operatorname{dom}\left(f \circ i_{G}\right)=G$ and $\operatorname{ran}\left(f \circ i_{G}\right)=f(G)$. Thus dom $\varphi\left(f \circ i_{G}\right)=h(G)$ and $\operatorname{ran} \varphi\left(f \circ i_{G}\right)=h(f(G))$. Now since $x \in G$ we have that $\varphi(f)(h(x)) \in h(f(G))$. Such sets $f(G)$ form a basis for the point $f(x)$ and so $\varphi(f)(h(x))=h(f(x))$. But this means that $\varphi(f)(y)=$ $\left(h \circ f \circ h^{-1}\right)(y)$. Thus $\varphi(f)=h \circ f \circ h^{-1}$.

The proof of the converse of the theorem is straightforward.

Thron (1962) has shown that two $T_{D}$-spaces (a weaker requirement than $\left.T_{1}\right) X$ and $Y$ are homeomorphic if and only if their lattices of closed subsets are lattice-equivalent. An isomorphism from $\mathscr{I}_{G}(X)$ onto $\mathscr{I}_{G}(Y)$ induces an equivalence between the lattices of open subsets (idempotents in $\mathscr{F}_{G}(X)$ are identity maps on open subsets) which in turn induces an equivalence between the lattices of closed subsets. Hence theorem 2.1 may be derived from Thron's result. Schein (1965) also has a similar result for differentiable manifolds. If $V^{\prime}$ is a differentiable manifold of class $C^{r}$ (where $r \geqq 0$ ) and $\operatorname{LDiff}^{p}\left(V^{r}\right.$ ) (where $p \leqq r)$ denotes the class of all local diffeomorphisms of class $C^{p}$ whose domain and range are open subsets of $V^{r}$ then $\operatorname{LDiff}^{p}\left(V^{r}\right)$ forms an inverse semigroup under composition. Schein's paper states that if $\Psi$ is an isomorphism from $\operatorname{LDiff}^{p}\left(V^{q}\right)$ onto $\operatorname{LDiff}^{p}\left(W^{r}\right)$ then there exists a diffeomorphism $h$ of class $C^{p}$ from the manifold $V^{q}$ onto the manifold $W^{r}$ such that $\Psi(f)=$ $h \circ f \circ h^{-1}$ for all $f \in \operatorname{LDiff}^{p}\left(V^{a}\right)$.

\section{Ideals of $\mathscr{I}_{G}(X)$}

If $S$ is an inverse semigroup and $\mathcal{U} \subseteq S$ then $\mathcal{U}$ is an ideal of $S$ if and only if $S \mathscr{U} S=\mathcal{U}$. If $S$ contains a zero element 0 , then $\{0\}$ will be an ideal of $S$. 
Now if $X$ is a discrete space then $\mathscr{I}_{G}(X)$ is just $\mathscr{I}_{X}$, the symmetric inverse semigroup on the set $X$, and so if $f$ and $g$ belong to $\mathscr{I}_{G}(X)$ then they will generate the same ideal of $\mathscr{I}_{C}(X)$ if and only if $\operatorname{dom} f$ and $\operatorname{dom} g$ have the same cardinality. Thus there are many ideals. The next results present the contrasting situation and show that for many spaces $X$ the semigroup $\mathscr{I}_{G}(X)$ is 0 -simple. Recall that all topological spaces are assumed to be nonempty.

THEOREM 3.1. $\mathscr{I}_{G}(X)$ is 0-simple if and only if every nonempty open subset of $X$ contains an open subset homeomorphic to $X$.

ProOF. Suppose $\mathscr{I}_{(}(X)$ is 0 -simple and $U$ is a nonempty open subset of $X$. Let $\mathcal{U}$ be the ideal generated by $i_{U}$. Then $\mathscr{U} \neq\{0\}$ and so $\mathscr{U}=\mathscr{I}_{G}(X)$. Hence $i_{X} \in \mathcal{U}$ and so $i_{X}=f \circ i_{l} \circ g$ for some $f, g \in \mathscr{I}_{G}(X)$. But then dom $g=$ $X$ and $g$ maps $X$ homeomorphically onto an open subset of $U$.

Now suppose that every nonempty open subset of $X$ contains an open subset homeomorphic to $X$. Let $\mathcal{U}$ be an ideal of $\mathscr{I}_{G}(X)$ with $\mathscr{U} \neq\{0\}$. Suppose $f \in \mathcal{U}$ and $f \neq 0$. Then if $U=\operatorname{dom} f$ we have that $i_{U} \in \mathcal{U}$ and $U \neq \varnothing$. By assumption $U$ contains an open subset $V$ which is homeomorphic to $X$. Let $h$ be a homeomorphism from $V$ onto $X$. Then $h \in \mathscr{I}_{G}(X)$ and so $h \circ i_{U} \circ h^{-1} \in \mathscr{U}$. But $h \circ i_{U} \circ h^{1}=i_{X}$ and so $\mathcal{U}=\mathscr{I}_{G}(X)$. Thus $\mathscr{I}_{G}(X)$ is 0 -simple.

COROLlaRY 3.2. $\mathscr{I}_{\mathrm{G}}\left(R^{n}\right)$ is 0-simple for $n$ finite.

COROllary 3.3. Let $Q$ denote the space of rational numbers. Then $\mathscr{I}_{G}(Q)$ is 0-simple.

COROllary 3.4. $\mathscr{I}_{G}(\mathscr{C})$ is 0 -simple where $\mathscr{C}$ is the Cantor discontinuum.

COROLl.ARY 3.5. If $\mathscr{I}_{G}(X)$ is 0 -simple and $Y$ is an open subset of $X$ then $\mathscr{I}_{G}(Y)$ is 0 -simple.

Proposition 3.6. Suppose $\mathscr{I}_{G}\left(X_{\alpha}\right)$ is 0 -simple for all $\alpha \in \Lambda$. Then $\mathscr{F}_{G}\left(\Pi_{\alpha \in}, X_{\alpha}\right)$ is 0 -simple.

Proof. Let $U$ be a nonempty open subset of $\Pi_{\alpha \in 1} X_{\alpha}$. Then there exist a finite number of distinct $\alpha_{i}, i=1 \cdots n$ and nonempty open sets $U_{\alpha_{i}} \subseteq X_{\alpha_{i}}$, $i=1 \cdots n$ such that

$$
p_{\alpha_{1}}^{-1}\left(U_{a_{1}}\right) \cap \cdots \cap p_{a_{n}}^{-1}\left(U_{\alpha_{n}}\right) \subseteq U
$$

where $p_{\alpha_{1}}$ is the projection map from $\Pi_{\alpha \in \in 1} X_{\alpha}$ onto $X_{\alpha_{t}}$. Since $\mathscr{F}_{G}\left(X_{\alpha_{t}}\right)$ is 0 -simple for each $i=1 \cdots n$ there exist open sets $V_{\alpha_{i}}$ and maps $h_{\alpha_{t}}$ such that $V_{\alpha_{i}} \subseteq U_{\alpha_{i}}$ and $h_{\alpha_{i}}$ is a homeomorphism from $V_{\alpha_{i}}$ onto $X_{\alpha_{i}}$ for $i=1 \cdots n$. Define a set $V$ in $\Pi_{\alpha \in 1} X_{\alpha}$ by 


$$
V=p_{\alpha_{1}}^{-1}\left(V_{\alpha_{1}}\right) \cap \cdots \cap p_{\alpha_{n}}^{-1}\left(V_{\alpha_{n}}\right)
$$

Then $V$ is open and $V \subseteq U$. Define a map $h$ from $V$ into $\Pi_{\alpha \in A_{1}} X_{\alpha}$ by

$$
(h(x))_{\alpha}=\left\{\begin{array}{l}
h_{\alpha_{i}}\left(x_{\alpha_{i}}\right) \text { if } \alpha=\alpha_{i} \text { for some } i=1 \cdots n \\
x_{\alpha} \text { otherwise. }
\end{array}\right.
$$

Then $h$ is a well defined map and it is straightforward to show that $h$ is a homeomorphism from $V$ onto $\Pi_{\alpha \in \Lambda} X_{\alpha}$. Now by theorem $3.1 \mathscr{I}_{G}\left(\Pi_{\alpha \in \Lambda} X_{\alpha}\right)$ is 0 -simple.

Corollary 3.7. Let $X$ be any open subset of $R^{n}$ ( $n$ not necessarily finite). Then $\mathscr{I}_{G}(X)$ is 0 -simple.

The discrete case and the spaces mentioned in the last few results are at opposite ends of the spectrum. When $X=I$, the closed unit interval, the semigroup $\mathscr{I}_{G}(I)$ is not 0 -simple and yet has very few ideals. Its ideals are enumerated below.

The ideal, in $\mathscr{I}_{G}(I)$, generated by the map $i_{(0,1)}$ is just the semigroup $\mathscr{I}_{G}(0,1)$. If $\mathcal{U}$ is the ideal generated by $i_{(0.1)}$ and $\mathscr{V}$ is the ideal generated by $i_{V}$ where $V=\left[0, \frac{1}{2}\right) \cup\left(\frac{1}{2}, 1\right]$ then

$$
\{0\} \subsetneq \mathscr{I}_{G}(0,1) \subsetneq \mathcal{U} \subsetneq \mathscr{V} \subsetneq \mathscr{I}_{G}(I) .
$$

Now let $f$ belong to $\mathscr{I}_{G}(I)$. We show that the ideal generated by $f$ is one of the ideals listed above. The maps $f$ and $i_{\text {dom } f}$ generate the same ideal. Let $G=\operatorname{dom} f$ and suppose $\mathcal{U}^{\prime}$ is the ideal generated by $i_{G}$. Assume $G \neq \varnothing$. If $G \subseteq(0,1)$ then $i_{G} \in \mathscr{I}_{G}(0,1)$ and if $f, h \in \mathscr{I}_{G}(I)$ then $f \circ i_{G} \circ h \in \mathscr{I}_{G}(0,1)$ also. Hence $U^{\prime} \subseteq \mathscr{I}_{G}(0,1)$. But if $G \subseteq(0,1)$ then $G$ contains an interval $(a, b)$ and so $i_{G}$ generates $\mathscr{I}_{G}(0,1)$. Thus $\mathscr{I}_{G}(0,1) \subseteq \mathcal{U}^{\prime}$ and so $\mathcal{U}^{\prime}=\mathscr{I}_{G}(0,1)$. If $0 \in G$ but $1 \notin G$ then $G$ contains a set $[0, a)$ which is homeomorphic to $(0,1]$. Thus $\mathcal{U} \subseteq \mathcal{U}^{\prime}$. Now $i_{(0,1)} \in \mathcal{U}$ since $[0,1)$ and $(1,0]$ are homeomorphic. But then $i_{G}=i_{G} \circ i_{[0,1)}$ and so $i_{G} \in \mathcal{U}$. Hence $\mathcal{U}^{\prime} \subseteq \mathcal{U}$ and so $\mathcal{U}^{\prime}=\mathcal{U}$. If $0 \in G$ and $1 \in G$ but $G \neq[0,1]$ then $G$ contains a set of the form $[0 \cdot a) \cup(b, 1]$ which is homeomorphic to $\left[0, \frac{1}{2}\right) \cup\left(\frac{1}{2}, 1\right]$. Thus $\mathscr{V} \subseteq \mathcal{U}^{\prime}$. But $G \subseteq[0, c) \cup(c, 1]$ for some $c$ and since this set is homeomorphic to $V$ we have that $i_{G} \in \mathscr{V}$. Hence $\mathcal{U}^{\prime} \subseteq \mathscr{V}$ and so $\mathscr{V}=\mathscr{U}^{\prime}$. This shows that any element in $\mathscr{I}_{G}(I)$ generates one of the listed ideals and since these ideals form a finite chain, any ideal in $\mathscr{I}_{G}(I)$ is of this form.

\section{Congruences on $\mathscr{I}_{G}(X)$}

In this section we look at various congruences on $\mathscr{I}_{G}(X)$. All spaces are assumed to be $T_{2}$. If $\rho$ is a congruence then $0_{\rho}$ will denote the set $\{f:(0, f) \in \rho\}$. The notation $\bar{U}$ means the closure of $U$. 
THEOREM 4.1. Let $\rho$ be a congruence on $\mathscr{F}_{G}(X)$ such that $0_{\rho}=\{0\}$. Suppose $(f, g) \in \rho$. Then $\overline{\operatorname{dom} f}=\overline{\operatorname{dom} g}$ and if $x \in \operatorname{dom} f \cap \operatorname{dom} g$ then $f(x)=$ $g(x)$.

Proof. Let $\rho$ be a congruence on $\mathscr{I}_{G}(X)$ with $0_{\rho}=\{0\}$ and suppose $f, g) \in \rho$. Let $y \in \overline{\operatorname{dom} f}$. If $y \notin \overline{\operatorname{dom} g}$ then there exists an open set $U$ such hat $y \in U$ but $U \cap \operatorname{dom} g=\varnothing$. Now $U \cap \operatorname{dom} f \neq \varnothing$ since $y \in \overline{\operatorname{dom} f}$. Hence $f \circ i_{U} \neq 0$. Since $(f, g) \in \rho$ we have that $\left(f \circ i_{U} . g \circ i_{U}\right) \in \rho$. But since $g \circ i_{U}=0$ his means that $f \circ i_{u} \in 0$, which is a contradiction. Hence $\overline{\operatorname{dom} f} \subseteq \overline{\operatorname{dom} g}$. Likewise $\overline{\operatorname{dom} g} \subseteq \overline{\operatorname{dom} f}$.

Now suppose $(f, g) \in \rho$ and $x \in \operatorname{dom} f \cap \operatorname{dom} g$. If $f(x) \neq g(x)$ then shoose open $U, V$ such that $f(x) \in U, g(x) \in V$ but $U \cap V=\varnothing$. Then $x \in f^{-1}(U) \cap g{ }^{\prime}(V)$ and if $G=f^{-1}(U) \cap g^{-1}(V)$ then $\left(i_{V} \circ f \circ i_{G}, i_{V} \circ g \circ i_{G i}\right)$ $\equiv \rho$. But $i_{V} \circ f \circ i_{i}=0$ and $i_{V} \circ g \circ i_{G} \neq 0$. This is a contradiction. Hence $f(x)=g(x)$.

TheOREM 4.2. Let $f, g \in \mathscr{I}_{G}(X)$. Define a relation $\rho_{1}$ on $\mathscr{I}_{G}(X)$ by 'f. $g) \in \rho_{1}$ if $\overline{\operatorname{dom} f}=\overline{\operatorname{dom} g}$ and for every $x \in \operatorname{dom} f \cap \operatorname{dom} g, f(x)=g(x)$. Then $\rho_{1}$ is a congruence.

Proof. Let $\rho_{1}$ be defined as above. First we show that $\rho_{\mathrm{I}}$ is an zquivalence relation. Clearly $(f, f) \in \rho_{1}$ for all $f \in \mathscr{I}_{G}(X)$ and if $(f, g) \in \rho_{1}$ then $i g, f) \in \rho_{1}$. Now suppose $(f, g) \in \rho_{1}$ and $(g, h) \in \rho_{1}$. Then $\overline{\operatorname{dom} f}=\overline{\operatorname{dom} g}$ and $\overline{\operatorname{dom} g}=\overline{\operatorname{dom} h}$. Hence $\overline{\operatorname{dom} f}=\overline{\operatorname{dom} h}$. If $x \in \operatorname{dom} f \cap \operatorname{dom} g \cap \operatorname{dom} h$ then $f(x)=g(x)$ since $(f, g) \in \rho_{1}$ and $g(x)=h(x)$ since $(g, h) \in \rho_{1}$. Hence $f(x)=$ $h(x)$. Since $X$ is $T_{2}$ and $f$ and $h$ agree on $\operatorname{dom} f \cap \operatorname{dom} g \cap \operatorname{dom} h$, then $f$ and $h$ agree on $\overline{\operatorname{dom} f \cap \operatorname{dom} g} \overline{\cap \operatorname{dom} h} \cap \operatorname{dom} f \cap \operatorname{dom} h$. Now let $x \in \operatorname{dom} f \cap$ Jom $h$. We must show that $f(x)=h(x)$. If $U$ is any open set containing $x$ then $U \cap \operatorname{dom} f \cap \operatorname{dom} h$ is an open set containing $x$. Since $x \in \operatorname{dom} f$ and $\overline{\operatorname{dom} f}=\overline{\operatorname{dom} g}$ we have $x \in \overline{\operatorname{dom} g}$. Hence $(U \cap \operatorname{dom} f \cap \operatorname{dom} h) \cap$ Jom $g \neq \varnothing$. This shows that any open set containing $x$ has nonempty intersection with $\operatorname{dom} f \cap \operatorname{dom} g \cap \operatorname{dom} h$. Thus $x \in \overline{\operatorname{dom} f \cap \operatorname{dom} g \cap \operatorname{dom} h}$ and by the above $f(x)=h(x)$. This means that $(f, h) \in \rho_{1}$ and so $\rho_{1}$ is an equivalence relation.

Next we show that $\rho_{1}$ is a congruence. Let $(f, g) \in \rho_{1}$ and $h \in \mathscr{I}_{G}(X)$. We will first show that $(f \circ h, g \circ h) \in \rho_{l}$. Now

$$
\begin{aligned}
& \overline{\operatorname{dom}(f \circ h)}=\overline{h^{\prime}(\operatorname{ran} h \cap \operatorname{dom} f)} \\
& \overline{\operatorname{dom}(g \circ h)}=\overline{h^{\prime}(\operatorname{ran} h \cap \operatorname{dom} g)} .
\end{aligned}
$$

Let $y \in h^{-1}(\operatorname{ran} h \cap \operatorname{dom} f)$ and let $V$ be any open subset of dom $h$ containing 
$y$. Then $h(y) \in \operatorname{ran} h \cap \operatorname{dom} f$ and $h(V)$ is an open set containing $h(y)$. Let $G=h(V) \cap \operatorname{dom} f$. Then $h(y) \in G$ and since $\overline{\operatorname{dom} f}=\overline{\operatorname{dom} g}$ we have that $G \cap \operatorname{dom} g \neq \varnothing$. Hence $G \cap \operatorname{ran} h \cap \operatorname{dom} g \neq \varnothing$ and $h^{-1}(G \cap \operatorname{ran} h \cap \operatorname{dom} g)$ $\neq \varnothing$. But $h^{-1}(G \cap \operatorname{ran} h \cap \operatorname{dom} g) \subseteq V$ and so $V \cap h^{-1}(\operatorname{ran} h \cap \operatorname{dom} g)$ $\neq \varnothing$. This means that $y \in \overline{h^{-1}(\operatorname{ran} h \cap \operatorname{dom} g)}$. Hence $h^{-1}(\operatorname{ran} h \cap \operatorname{dom} f) \subset$ $\overline{h^{-1}(\operatorname{ran} h \cap \operatorname{dom} g)}$. But then $\operatorname{dom}(f \circ h) \subseteq \overline{\operatorname{dom}(g \circ h)}$ and so $\overline{\operatorname{dom}(f \circ h) \subseteq}$ $\overline{\operatorname{dom}(g \circ h)}$. The exact same method shows that $\overline{\operatorname{dom}(g \circ h)} \subseteq \overline{\operatorname{dom}(f \circ h)}$. Thus $\overline{\operatorname{dom}(f \circ h)}=\overline{\operatorname{dom}(g \circ h)}$. Now let $x \in \operatorname{dom}(f \circ h) \cap \operatorname{dom}(g \circ h)$. Then $x \in \operatorname{dom} h$ and $h(x) \in \operatorname{dom} f \cap \operatorname{dom} g$. Since $(f, g) \in \rho_{1}$ this means that $f(h(x))=g(h(x))$ and so $(f \circ h, g \circ h) \in \rho_{1}$.

Finally we consider $h \circ f$ and $h \circ g$. We have that

$$
\begin{aligned}
& \overline{\operatorname{dom}(h \circ f)}=\overline{f^{-1}(\operatorname{dom} h \cap \operatorname{ran} f)} \\
& \overline{\operatorname{dom}(h \circ g)}=\overline{g^{-1}(\operatorname{dom} h \cap \operatorname{ran} g)} .
\end{aligned}
$$

Let $x \in f^{-1}(\operatorname{dom} h \cap \operatorname{ran} f)$ and suppose $U$ is an open subset of $\operatorname{dom} f$ containing $x$. Then $f(x) \in \operatorname{dom} h$. Let $V=f(U) \cap \operatorname{dom} h$. Then $V$ is open and $f(x) \in V$. Now $f^{-1}(V)$ is an open set containing $x$ and so $f^{-1}(V) \cap \operatorname{dom} g \neq \varnothing$ $(\overline{\operatorname{dom} f}=\overline{\operatorname{dom} g}, x \in \operatorname{dom} f)$. Let $G=f^{-1}(V) \cap \operatorname{dom} g$. Then $G \subseteq U, G \neq \varnothing$ and $G \subset \operatorname{dom} f \cap \operatorname{dom} g$. This means that $f(G)=g(G)$. Hence $g(G) \subseteq V \subseteq$ dom $h$. But then $g(G) \subseteq \operatorname{dom} h \cap$ ran $g$ and so $G \subseteq g^{-1}$ (dom $h \cap \operatorname{ran} g$ ). But

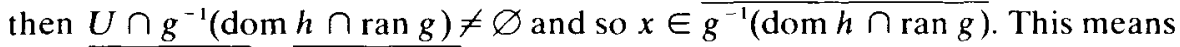

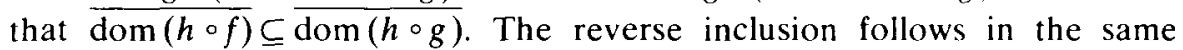
manner and hence $\overline{\operatorname{dom}(h \circ f)}=\overline{\operatorname{dom}(h \circ g)}$. Now if $x \in \operatorname{dom}(h \circ f) \cap$ $\operatorname{dom}(h \circ g)$ then $x \in \operatorname{dom} f \cap \operatorname{dom} g$ and so $f(x)=g(x)$. But then $h(f(x))=$ $h(g(x))$ and hence $(h \circ f, h \circ g) \in \rho_{1}$. This completes the proof that $\rho_{1}$ is a congruence.

Corollary 4.3. Let $\rho$ be a congruence on $\mathscr{I}_{G}(X)$. Then $0_{\rho}=\{0\}$ if and only if $\rho \subseteq \rho_{1}$.

Proof. Suppose $\rho$ is a congruence and $0_{\rho}=\{0\}$. Then by theorem 4.1 we have that if $(f, g) \in \rho$ then $(f, g) \in \rho_{1}$. Conversely, suppose $\rho \subseteq \rho_{1}$. Let $(f, 0) \in \rho$. Then $(f, 0) \in \rho_{1}$ and so $\overline{\operatorname{dom} f}=\varnothing$. Hence $f=0$ and $0_{\rho}=\{0\}$.

Corollary 4.4. Suppose $\mathscr{I}_{G}(X)$ is 0 -simple and $\rho$ is a proper congruence on $\mathscr{I}_{G}(X)$. Then $\rho \subseteq \rho_{\mathrm{I}}$.

Proof. If $\rho$ is a congruence on $\mathscr{I}_{G}(X)$ then since $0_{\rho}$ is always an ideal of $\mathscr{I}_{G}(X)$ and $\mathscr{I}_{G}(X)$ is 0 -simple we have that $0_{\rho}=\mathscr{I}_{G}(X)$ or $0_{\rho}=\{0\}$. If $\rho$ is a proper congruence then $0_{\rho} \neq \mathscr{I}_{G}(X)$ and so $0_{\rho}=\{0\}$. But then $\rho \subseteq \rho_{1}$ by the last corollary. 
In the last section we showed that for many spaces $X, \mathscr{F}_{G}(X)$ is 0 -simple. Recall that $R^{n}$ denotes the Cartesian product of $n$ copies of the real line ( $n$ nay be infinite), $Q$ denotes the space of rational numbers, and $\mathscr{C}$ denotes the Zantor discontinuum. The last corollary then yields:

CoRollary 4.5. $\rho_{1}$ is the largest proper congruence on $\mathscr{I}_{G}\left(R^{n}\right)$, on $\mathscr{F}_{G}(Q)$ and on $\mathscr{F}_{G}(\mathscr{C})$.

When $X$ is a discrete space $\rho_{1}=i$ (the diagonal congruence) since if $\overline{\operatorname{lom} f}=\overline{\operatorname{dom} g}$ then $\operatorname{dom} f=\operatorname{dom} g$ and so if $(f, g) \in \rho_{\text {। }}$ then $f=g$. If $X$ is a discrete space and $\rho$ is a congruence on $\mathscr{I}_{G}(X)$ such that $0_{\rho}=\{0\}$ then $\rho \subseteq \rho_{1}$, sy corollary 4.3 and so $\rho$ is the diagonal congruence. In general, the :ongruence $\rho_{1}$ does not separate idempotents. In fact, if $\rho_{1}$ separates dempotents then $X$ is discrete. This result stems from the following general heorem which proves that the only idempotent separating congruence on $\mathscr{f}_{G}(X)$ is the diagonal congruence.

THEOREM 4.6. Let $\rho$ be a congruence on $\mathscr{I}_{G}(X)$ which separates idempotents. Then $\rho$ is the diagonal congruence.

Proof. Suppose $\rho$ is a congruence which separates idempotents. First we show that $0_{\rho}=\{0\}$. If $(0, h) \in \rho$ then $\left(0, h^{-1} \circ h\right) \in \rho$. Since $\rho$ separates dempotents this means that $h=0$ and $0_{\rho}=\{0\}$. Now suppose $\rho$ is not the diagonal congruence. Then there exist $f, g \in \mathscr{I}_{G}(X)$ such that $(f, g) \in \rho$ but $f \neq g$. Since $0_{p}=\{0\}$ we can apply thoerem 4.1 to conclude that $\overline{\operatorname{dom} f}=\overline{\operatorname{dom} g}$ and $f(x)=g(x)$ for all $x \in \operatorname{dom} f \cap \operatorname{dom} g$. Since $f \neq g$ but $f$ and $g$ agree on their common domain we must have that $\operatorname{dom} f \neq \operatorname{dom} g$. Set $\operatorname{dom} f=U$ and

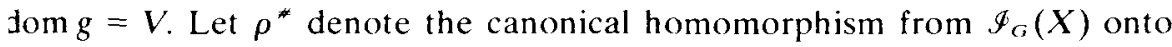
$\mathscr{\Psi}_{G}(X) / \rho$. Since $(f, g) \in \rho$ we have that $\rho^{*}(f)=\rho^{*}(g)$ and hence $\rho^{*}\left(f^{-1}\right)=$ $\rho^{*}\left(g^{-1}\right)$. But then

$$
\begin{aligned}
\rho^{*}\left(i_{U}\right) & =\rho^{*}\left(f^{-1} \circ f\right)=\rho^{*}\left(f^{-1}\right) \rho^{*}(f)=\rho^{*}\left(g^{-1}\right) \rho^{*}(g) \\
& =\rho^{*}\left(g^{-1} \circ g\right)=\rho^{*}\left(i_{V}\right) .
\end{aligned}
$$

This means that $\left(i_{U}, i_{v}\right) \in \rho$ which is a contradiction since $\rho$ separates idempotents and $U \neq V$. Hence $\rho$ is the diagonal congruence.

THEOREM 4.7. The following are equivalent for $\mathscr{I}_{G}(X)$ :

1) $\rho_{1}$ separates idempotents.

2) $\rho_{1}$ is the diagonal congruence.

3) $X$ is discrete.

ProOF. 1) implies 2) is a consequence of the last theorem. We now show that 2) implies 3). If $|X|=1$ then trivially $X$ is discrete. Suppose $|X|>1$ and 
$x \in X$. Since $X$ is $T_{1}$ the set $X-\{x\}$ is open. If $X-\{x\}$ is not closed also then $\overline{X-\{x\}}=X$. But then $\overline{\operatorname{dom} i_{X-\{x\}}}=\overline{\operatorname{dom} i_{X}}$ and $i_{X-\{x\}}$ and $i_{X}$ agree on their common domain. Hence $\left(i_{x}\{x\}, i_{x}\right) \in \rho_{1}$. But we are assuming that $\rho_{1}$ is the diagonal congruence. This is a contradiction. Thus the set $X-\{x\}$ must be closed. But then the set $\{x\}$ is open and $X$ is discrete.

We now show that 3) implies 1). Suppose $X$ is discrete and $(f, g) \in \rho_{1}$. Then $\operatorname{dom} f=\overline{\operatorname{dom} f}=\overline{\operatorname{dom} g}=\operatorname{dom} g$ and $f(x)=g(x)$ for all $x \in \operatorname{dom} f \cap$ dom $g$. Hence $f=g$ and $\rho_{1}$ is the diagonal congruence and so certainly $\rho_{1}$ separates idempotents.

If $X$ is a discrete space then, as remarked earlier, $\mathscr{I}_{G}(X)$ is just the inverse semigroup $\mathscr{I}_{X}$ of all partial injective maps on the set $X$. Congruences on $\mathscr{I}_{X}$ have been studied (see Scheiblich (1973)). If $X$ is not a discrete space then by the last theorem $\rho_{1}$ is not the diagonal congruence. Clearly $\rho_{1}$ is not the universal congruence nor a Rees congruence $\left(0_{\rho_{1}}=\{0\}\right)$. The next few results will be used to determine another congruence $\rho_{*}$ on $\mathscr{I}_{G}(X)$ and gain some information about it. We will use the notation $U \stackrel{*}{=} V$ if $U$ and $V$ differ by at most a finite number of points.

Definition 4.8. (Reilly \& Scheiblich (1967)). Let $S$ be an inverse semigroup and $P=\left\{E_{\alpha} ; \alpha \in J\right\}$ be a partition of the idempotents of $S$. Then $P$ is a normal partition if

1) $\alpha, \beta \in J$ implies that there exists $\gamma \in J$ such that $E_{\alpha} E_{\beta} \subseteq E_{\gamma}$.

2) $\alpha \in J$ and $f \in S$ implies that there exists $\beta \in J$ such that $f^{-1} E_{\alpha} f \subseteq E_{\beta}$.

THEOREM 4.9. (Reilly \& Scheibich (1967)). Let $P=\left\{E_{\alpha}: \alpha \in J\right\}$ be a normal partition of the idempotents of an inverse semigroup $S$. Let $\sigma_{1}=$ $\left\{(f, g) \in S \times S\right.$ : there exists $\alpha \in J$ with $f^{-1} f, g^{-1} g \in E_{\alpha}$ and, for some $e \in E_{\alpha}$, $f e=g e\}$ and $\sigma_{2}=\left\{(f, g) \in S \times S: \alpha \in J\right.$ implies that, for some $\beta \in J, f^{-1} E_{\alpha} f$, $\left.g^{-1} E_{\alpha} g \subseteq E_{\beta}\right\}$. Then $\sigma_{1}$ and $\sigma_{2}$ are, respectively, the smallest and largest congruences on $S$ which induce the partition $P$ of idempotents.

THEOREM 4.10. (Preston (1954)). Let $\rho$ be a congruence on an inverse semigroup $S$. Then $\rho$ is a congruence on the idempotents of $S$ and the partition (into equivalence classes) that $\rho$ induces is a normal partition.

THEOREM 4.11. Let $f, g \in \mathscr{I}_{G}(X)$. Define a relation $\rho_{2}$ on $\mathscr{I}_{G}(X)$ by $(f, g) \in \rho_{2}$ if $\operatorname{dom} f \stackrel{*}{=} \operatorname{dom} g$ and $|\{x: x \in \operatorname{dom} f \cap \operatorname{dom} g, f(x) \neq g(x)\}|<\mathbf{N}_{0}$. Then $\rho_{2}$ is a congruence on $\mathscr{I}_{G}(X)$. Furthermore, if $\rho$ is a congruence on $\mathscr{I}_{G}(X)$ which induces the same partition of idempotents that $\rho_{2}$ does, then $\rho_{2} \subseteq \rho$.

Proof. Define a relation $\sigma$ on the idempotents of $\mathscr{I}_{G}(X)$ by $\left(i_{U}, i_{v}\right) \in \sigma$ 
if $U \stackrel{*}{=} V$. Then $\sigma$ is an equivalence relation and induces a partition $P=$ $\left\{E_{U}: U\right.$ open in $\left.X\right\}$ where $E_{U}=\left\{i_{V}: U \stackrel{*}{=} V\right\}$. We show that $P$ is a normal partition. Let $E_{U}, E_{V} \in P$. Then $E_{U} \circ E_{V} \subseteq E_{u}$. If $f \in \mathscr{I}_{G}(X)$ then we will show that $f^{-1} \circ E_{U} \circ f \subseteq E_{f}{ }^{\prime}(u)$. Suppose $i_{v} \in E_{l}$. Then $f^{-1} \circ i_{v} \circ f=i_{f}{ }^{\prime}(v)$. But $i_{f^{-1}(v)} \in E_{f}{ }^{\prime}(U)$ since $U \stackrel{*}{=} V$. Hence $f^{-1} \circ E_{U^{\prime}} \circ f \subseteq E_{f^{-1}(U)}$ and $P$ is a normal partition.

According to theorem $4.9 P$ has associated with it a smallest congruence $\sigma_{1}=\left\{(f, g): f^{-1} \circ f, g^{-1} \circ g \in E_{U}\right.$ for some $E_{U} \in P$ and $f \circ i_{V}=g \circ i_{V}$ for some $\left.i_{1} \in E_{U}\right\}$. But this just says that $\operatorname{dom} f \stackrel{*}{=} \operatorname{dom} g$ and $\mid\{x: x \in \operatorname{dom} f \cap$ dom $g, f(x) \neq g(x)\} \mid<N_{0}$. Hence $\rho_{2}=\sigma_{1}$, and $\rho_{2}$ is a congruence which is the smallest congruence inducing the partition $P$ of idempotents.

Note that in most cases the congruences $\rho_{1}$ and $\rho_{2}$ are distinct. In fact, if $X$ has an open dense subset $D$ such that $|X-D| \geqq \mathcal{N}_{0}$ then $\rho_{1} \neq \rho_{2}\left(\left(i_{D}, i_{X}\right) \in \rho_{1}\right.$, but $\left.\left(i_{D}, i_{X}\right) \notin \rho_{2}\right)$. If $X$ is a finite space then $X$ is discrete and $\rho_{2}$ is the universal congruence. On the other hand, if $X$ is infinite then $\rho_{2}$ is a proper congruence. If $\mathscr{F}_{G}(X)$ is 0 -simple then $\rho_{1}$ is the largest proper congruence on $\mathscr{F}_{G}(X)$. In some instances the congruence $\rho_{2}$ is a minimal congruence on $\mathscr{H}_{G}(X)$.

THForem 4.12. Suppose $X$ and $\mathscr{I}_{G}(X)$ satisfy the following two conditions:

1) $\mathscr{I}_{i_{i}}(X)$ is 0 -simple.

2) $|X|>1$ and every open subset $U$ of $X$ is homogeneous (if $a, b \in U$ then there exists a homeomorphism $h$ from $U$ onto $U$ such that $h(a)=b)$. Then $\rho_{2}$ is a minimal proper congruence on $\mathscr{I}_{G}(X)$ (if $\rho$ is a congruence on $\mathscr{I}_{G_{3}}(X)$ and $\rho \subseteq \rho_{2}$ then either $\rho=\rho_{2}$ or $\rho$ is the diagonal congruence).

Proof. Suppose $X$ and $\mathscr{I}_{c}(X)$ satisfy the conditions of the theorem. Since $|X|>1$ and $\mathscr{F}_{G}(X)$ is 0 -simple we have that $\rho_{2}$ is not the diagonal congruence and using corollary 4.4 we have that $\rho_{2} \subseteq \rho_{1}$ and so $0_{p}=\{0\}$. This means that $\rho_{2}$ is not the universal congruence and so is a proper congruence.

Now suppose $\rho$ is a congruence on $\mathscr{I}_{i}(X), \rho$ is not the diagonal congruence and $\rho \subseteq \rho_{2}$. We will eventually prove that $\rho_{2} \subseteq \rho$. Since $\rho$ is not the diagonal congruence there exist $f . g \in \mathscr{F}_{\mathrm{i}}(X)$ such that $(f, g) \in \rho$ but $f \neq g$. This means that $\left(i_{\text {dimf }}, i_{\text {skmg }}\right) \in \rho$ (see proof of theorem 4.6). Now since $\mathscr{I}_{(i}(X)$ is 0 -simple and $|X|>1$ we get that $\rho \subseteq \rho_{2} \subseteq \rho_{1}$ and hence $f$ and $g$ agree on their common domain. But $f \neq g$ and so $\operatorname{dom} f \neq \operatorname{dom} g$. Let $W=\operatorname{dom} f$ and $V=\operatorname{dom} g$. Then $\left(i_{w}, i_{1}\right) \in \rho, W \neq V$ and $\bar{W}=\bar{V}$. Hence $|W| \geqq \boldsymbol{\aleph}_{i}$. Since $\rho \subseteq \rho_{2}$ we have that $W \stackrel{*}{=} V$. Now $\left(i_{1}, i_{w}\right) \in \rho$ and so $\left(i_{v}, i_{v} \cap w\right) \in \rho$ and $\left(i_{u}, i_{1}, u\right) \in \rho$ also. Since $V \neq W$ we have that $V \cap W \neq V$ or $V \cap W \neq W$. Without loss of generality suppose that $V \cap W \neq V$. Since $V \stackrel{*}{=} W$ this means that $V=(V \cap W) \cup\left\{x_{1}, \cdots, x_{n}\right\}$ where $n \geqq 1, n$ is finite and $x_{i} \notin(V \cap W)$ for 
$i=1 \cdots n$. Now since $V$ and $V \cap W$ are open we have that the set $G=\left((V \cap W) \cup\left\{x_{1}\right\}\right)$ is open. But since $V \cap W \subseteq G \subseteq V$ and $\left(i_{V}, i_{V \cap W}\right) \in \rho$ we get that $\left(i_{G}, i_{V \cap W}\right) \in \rho$. Hence we have a nonempty infinite open set $G$ such that $\left(i_{G}, i_{G-\left\{x_{1}\right\}}\right) \in \rho$ where $x_{1} \in G$. Now let $y \in G$. Since $G$ is homogeneous there exists a homeomorphism $h$ from $G$ onto $G$ such that $h\left(x_{1}\right)=y$. But then $\left(h \circ i_{G} \circ h^{-1}, h \circ i_{G-\left\{x_{1}\right\}} \circ h^{-1}\right) \in \rho$ which means that $\left(i_{G}, i_{G-\{y\}}\right) \in \rho$. If $y, z \in$ $G, y \neq z$ then $\left(i_{G} \circ i_{G-\{z\}}, i_{G-\{y\}} \circ i_{G,\{z\}}\right) \in \rho$ and hence $\left(i_{G ;\{z\}}, i_{G-\{z, y\}}\right) \in \rho$. Since $\left(i_{G}, i_{G-\{z\}}\right) \in \rho$ this means that $\left(i_{G}, i_{G-\{z . y\}}\right) \in \rho$. We can continue this process to obtain the result that if $F$ is a finite subset of $G$ then $\left(i_{G}, i_{G-F}\right) \in \rho$.

Let $K$ be any finite subset of $X$. We will show that $\left(i_{X}, i_{X-K}\right) \in \rho$. Since $\mathscr{I}_{G}(X)$ is 0 -simple $G$ contains an open subset $G^{\prime}$ which is homeomorphic to $X$. Let $f$ map $G^{\prime}$ homeomorphically onto $X$ and let $F=f^{-1}(K)$. Then $F$ is a finite subset of $G$ and so $\left(i_{G}, i_{G-F}\right) \in \rho$ and so $\left(f \circ i_{G} \circ f^{-1}, f \circ i_{G-F}, f^{-1}\right) \in \rho$. But this means that $\left(i_{X}, i_{X-K}\right) \in \rho$.

Now let $U$ be an open subset of $X$ and let $F$ be any finite subset of $U$. Then $\left(i_{X} \circ i_{U}, i_{X-F} \circ i_{U}\right) \in \rho$ and so $\left(i_{U}, i_{U-F}\right) \in \rho$. We finally show that $\rho$ and $\rho_{2}$ induce the same partition of idempotents. If $\left(i_{U}, i_{V}\right) \in \rho$ then $\left(i_{v}, i_{V}\right) \in \rho_{2}$ since $\rho \subseteq \rho_{2}$. Conversely, if $\left(i_{U}, i_{V}\right) \in \rho_{2}$ then $U \stackrel{*}{=} V$ and $\bar{U}=\bar{V}\left(\mathscr{F}_{G}(X)\right.$ is 0 -simple and $|X|>1$ means that $\left.\rho_{2} \subseteq \rho_{1}\right)$. Then $\overline{U \cup V}=\bar{U}$ and $U \cup V \stackrel{*}{=} U$. This means that $\left(i_{U \cup V}, i_{U}\right) \in \rho_{2}$ also. But then $U=(U \cup V)-F$ where $F$ is a finite subset of $U \cup V$ and so $\left(i_{U \cup V}, i_{U}\right) \in \rho$. Likewise $\left(i_{U \cup V}, i_{V}\right) \in \rho$ and so $\left(i_{U}, i_{V}\right) \in$ $\rho$. Thus $\left(i_{U}, i_{V}\right) \in \rho$ if and only if $\left(i_{U}, i_{V}\right) \in \rho_{2}$. Hence $\rho$ and $\rho_{2}$ induce the same partition of idempotents of $\mathscr{I}_{G}(X)$. Now by theorem $4.11 \rho_{2} \subseteq \rho$. But this means that $\rho_{2}=\rho$ and completes the proof.

COROLlaRY 4.13. The congruence $\rho_{2}$ is a minimal proper congruence on $\mathscr{I}_{G}(R), \mathscr{I}_{G}(Q)$, and $\mathscr{I}_{G}(\mathscr{C})$.

\section{References}

A. H. Clifford and G. B. Preston (1961, 1967), The Algebraic Theory of Semigroups, vol. 1 and II (American Math. Soc.).

G. B. Preston (1954), Inverse semigroups, J. London Math. Soc. 29, 396-403.

N. R. Reilly and H. E. Scheiblich (1967), Congruences on regular semigroups, Pacific J. of Math. 23, 349-360.

H. E. Scheiblich (1973), Concerning congruences on symmetric inverse semigroups, Czechoslovak Math. J. 23, 1-10.

B. M. Schein (1965), Materials of the Second Baltic Geometric Conference, Tartu, 179-182.

W. J. Thron (1962), Lattice-equivalence of topological spaces. Duke Mathematical J. 29, 671-679.

Department of Mathematics,

University of Florida,

Gainesville, Florida,

U.S.A. 